Pacific

Journal of

Mathematics

PROPERTIES OF THE RESIDUAL CIRCLE ACTION ON A HYPERTORIC VARIETY

Megumi Harada And Nicholas Proudfoot

Volume $214 \quad$ No. 2

April 2004 


\title{
PROPERTIES OF THE RESIDUAL CIRCLE ACTION ON A HYPERTORIC VARIETY
}

\author{
Megumi Harada and Nicholas Proudfoot
}

\begin{abstract}
We consider an orbifold $X$ obtained by a Kähler reduction of $\mathbb{C}^{n}$, and we define its "hyperkähler analogue" $M$ as a hyperkähler reduction of $T^{*} \mathbb{C}^{n} \cong \mathbb{H}^{n}$ by the same group. In the case where the group is abelian and $X$ is a toric variety, $M$ is a toric hyperkähler orbifold, as defined in Bielawski and Dancer, 2000, and further studied by Konno and by Hausel and Sturmfels. The variety $M$ carries a natural action of $S^{1}$, induced by the scalar action of $S^{1}$ on the fibers of $T^{*} \mathbb{C}^{n}$. In this paper we study this action, computing its fixed points and its equivariant cohomology. As an application, we use the associated $\mathbb{Z}_{2}$ action on the real locus of $M$ to compute a deformation of the Orlik-Solomon algebra of a smooth, real hyperplane arrangement $\mathcal{H}$, depending nontrivially on the affine structure of the arrangement. This deformation is given by the $\mathbb{Z}_{2}$-equivariant cohomology of the complement of the complexification of $\mathcal{H}$, where $\mathbb{Z}_{2}$ acts by complex conjugation.
\end{abstract}

In order to construct a toric variety as a Kähler quotient of $\mathbb{C}^{n}$ by a torus, one begins with the combinatorial data of an arrangement $\mathcal{H}$ of $n$ cooriented, rational, affine hyperplanes in $\mathbb{R}^{d}$. The normal vectors to these hyperplanes determine a subtorus $T^{k} \subseteq T^{n}(k=n-d)$, and the affine structure determines a value $\alpha \in\left(\mathfrak{t}^{k}\right)^{*}$ at which to reduce, so that we may define $X=\mathbb{C}^{n} /{ }_{\alpha} T^{k}$. Using the same combinatorial data, one can also construct a hypertoric variety ${ }^{1}$ which is defined as the hyperkähler quotient $M=\mathbb{H}^{n} / \| /{ }_{(\alpha, 0)} T^{k}$ of $\mathbb{H}^{n} \cong T^{*} \mathbb{C}^{n}$ by the induced action of the same subtorus $T^{k} \subseteq T^{n}[\mathbf{B D}]$. It is well-known that the toric variety $X$ does not retain all of the information of $\mathcal{H}$; indeed, it depends only on the polyhedron $\Delta$ obtained by intersecting the half-spaces associated to each of the cooriented hyperplanes. Thus it is always possible to add an extra hyperplane to $\mathcal{H}$ without changing $X$. In contrast, the hypertoric variety $M$ remembers the number of hyperplanes in $\mathcal{H}$, but its equivariant diffeomorphism type depends neither on the coorientations nor on the affine structure of $\mathcal{H}$ (see Theorem 4.1, and Lemmas 2.1 and 2.2).

\footnotetext{
${ }^{1} \mathrm{In}[\mathrm{BD}, \mathbf{K} 1, \mathbf{K 2}]$ and [HS] $M$ is called a "toric hyperkähler" variety, but as it is a complex variety that is not toric in the standard sense, we prefer the term "hypertoric."
} 
The purpose of this paper is to study the hamiltonian $S^{1}$ action on $M$ descending from the scalar action of $S^{1}$ on the fibers of $T^{*} \mathbb{C}^{n}$. This action is sensitive to both the coorientations and the affine structure of $\mathcal{H}$, even on the level of equivariant cohomology (Section 4). One can recover the toric variety $X$ as the minimum of the $S^{1}$ moment map, hence the geometric structure of $M$ along with its circle action carries strictly more information than either $X$ or $M$ alone. In Section 3 we give an explicit description of this action when restricted to the core $C$, a deformation retract of $M$ which is a union of projective subvarieties. In Section 4 we compute the $S^{1}$ and $T^{d} \times S^{1}$-equivariant cohomologies of $M$, using the full combinatorial data of $\mathcal{H}$.

In Section 5, we examine the real locus $M_{\mathbb{R}} \subseteq M$, i.e., the fixed point set of an involution of $M$ that is anti-holomorphic with respect to the first complex structure. By studying the topology of $M_{\mathbb{R}}$, we interpret the results of Section 4 in terms of the Orlik-Solomon algebra $\mathcal{O S}=H^{*}(\mathcal{M}(\mathcal{H}))$, where $\mathcal{M}(\mathcal{H})$ is the complement of the complexification of $\mathcal{H}$. We show how to interpret Theorem 4.4 as a computation of $H_{\mathbb{Z}_{2}}^{*}\left(\mathcal{M}(\mathcal{H}) ; \mathbb{Z}_{2}\right)$, a deformation of the Orlik-Solomon algebra of a smooth, real arrangement that depends nontrivially on the affine structure. ${ }^{2}$

\section{Hyperkähler reductions.}

A hyperkähler manifold is a smooth manifold, necessarily of real dimension $4 n$, which admits three complex structures $J_{1}, J_{2}, J_{3}$ satisfying the usual quaternionic relations, in a manner compatible with a metric. Just as in the Kähler case, we can define three different symplectic forms on $N$ as follows:

$$
\omega_{1}(v, w)=g\left(J_{1} v, w\right), \omega_{2}(v, w)=g\left(J_{2} v, w\right), \omega_{3}(v, w)=g\left(J_{3} v, w\right) .
$$

Note that the complex-valued two-form $\omega_{2}+i \omega_{3}$ is nondegenerate and covariant constant, hence closed and holomorphic with respect to the complex structure $J_{1}$. Any hyperkäher manifold can therefore be considered as a holomorphic symplectic manifold with complex structure $J_{1}$, real symplectic form $\omega_{\mathbb{R}}:=\omega_{1}$, and holomorphic symplectic form $\omega_{\mathbb{C}}:=\omega_{2}+i \omega_{3}$. This is the point of view that we will adopt in this paper.

We will refer to an action of $G$ on a hyperkähler manifold $N$ as hyperhamiltonian if it is hamiltonian with respect to $\omega_{\mathbb{R}}$ and holomorphic hamiltonian with respect to $\omega_{\mathbb{C}}$, with $G$-equivariant moment map

$$
\mu_{\mathrm{HK}}:=\mu_{\mathbb{R}} \oplus \mu_{\mathbb{C}}: N \rightarrow \mathfrak{g}^{*} \oplus \mathfrak{g}_{\mathbb{C}}^{*}
$$

Theorem 1.1 ([HKLR]). Let $\left(N^{4 n}, g\right)$ be a hyperkähler manifold with real symplectic form $\omega_{\mathbb{R}}$ and holomorphic symplectic form $\omega_{\mathbb{C}}$. Suppose that $N$

${ }^{2}$ A more general computation of $H_{\mathbb{Z}_{2}}^{*}\left(\mathcal{M}(\mathcal{H}) ; \mathbb{Z}_{2}\right)$, in which $\mathcal{H}$ is not assumed to be simple, rational, or smooth, will appear in $[\mathbf{P r}]$. 
is equipped with a hyperhamiltonian action of a compact Lie group $G$, with moment map $\mu_{H K}=\mu_{\mathbb{R}} \oplus \mu_{\mathbb{C}}$. Suppose $\xi=\xi_{\mathbb{R}} \oplus \xi_{\mathbb{C}}$ is a central regular value of $\mu_{H K}$. Then there is a unique hyperkähler structure on the hyperkähler quotient $M=\left.N\|/\|\right|_{\xi} G:=\mu_{H K}^{-1}(\xi) / G$, with associated symplectic and holomorphic symplectic forms $\omega_{\mathbb{R}}^{\xi}$ and $\omega_{\mathbb{C}}^{\xi}$, such that $\omega_{\mathbb{R}}^{\xi}$ and $\omega_{\mathbb{C}}^{\xi}$ pull back to the restrictions of $\omega_{\mathbb{R}}$ and $\omega_{\mathbb{C}}$ to $\mu_{H K}^{-1}(\xi)$.

If $\xi \in \mathfrak{g}^{*} \oplus \mathfrak{g}_{\mathbb{C}}^{*}$ is fixed by the coadjoint action of $G$, the inverse image $\mu_{\mathbb{C}}^{-1}\left(\xi_{\mathbb{C}}\right)$ is preserved by $G$, and is a (singular) Kähler subvariety with respect to $\omega_{\mathbb{R}}$. Then by $[\mathbf{H L}]$ (see also $[\mathbf{N a}, 3.2]$ and $[\mathbf{S j}, 2.5]$ ), we have

$$
N / / /\left.\right|_{\xi} G=\mu_{\mathbb{C}}^{-1}\left(\xi_{\mathbb{C}}\right) / /_{\xi_{\mathbb{R}}} G=\mu_{\mathbb{C}}^{-1}\left(\xi_{\mathbb{C}}\right)^{\mathrm{ss}} / G_{\mathbb{C}}
$$

where

$$
\mu_{\mathbb{C}}^{-1}\left(\xi_{\mathbb{C}}\right)^{\mathrm{ss}}=\left\{x \in \mu_{\mathbb{C}}^{-1}\left(\xi_{\mathbb{C}}\right) \mid G x \cap \mu_{\mathbb{R}}^{-1}\left(\xi_{\mathbb{R}}\right) \neq \emptyset\right\} .
$$

We now specialize to the case where $G$ is a compact Lie group acting linearly on $\mathbb{C}^{n}$ with moment map $\mu: \mathbb{C}^{n} \rightarrow \mathfrak{g}^{*}$, taking $0 \in \mathbb{C}^{n}$ to $0 \in \mathfrak{g}^{*}$. This action induces an action of $G$ on the holomorphic cotangent bundle $T^{*} \mathbb{C}^{n} \cong \mathbb{C}^{n} \times\left(\mathbb{C}^{n}\right)^{*}$. If we choose a bilinear inner product on $\mathbb{C}^{n}$, we can coordinatize this representation as $\left\{(z, w) \mid z, w \in \mathbb{C}^{n}\right\}$ with $g(z, w)=$ $\left(g z, g^{-1} w\right)$. Choose an identification of $\mathbb{H}^{n}$ with $T^{*} \mathbb{C}^{n}$ such that the complex structure $J_{1}$ on $\mathbb{H}^{n}$ given by right multiplication by $i$ corresponds to the natural complex structure on $T^{*} \mathbb{C}^{n}$. Then $T^{*} \mathbb{C}^{n}$ inherits a hyperkähler, and therefore also a holomorphic symplectic, structure, with $\omega_{\mathbb{R}}$ given by adding the standard symplectic structures on $\mathbb{C}^{n}$ and $\left(\mathbb{C}^{n}\right)^{*} \cong \mathbb{C}^{n}$, and $\omega_{\mathbb{C}}=d \eta$, where $\eta$ is the canonical holomorphic 1 -form on $T^{*} \mathbb{C}^{n}$.

Note that $G$ acts $\mathbb{H}$-linearly on $T^{*} \mathbb{C}^{n} \cong \mathbb{H}^{n}$ (where $n \times n$ matrices act on the left on $\mathbb{H}^{n}$, and scalar multiplication by $\mathbb{H}$ is on the right), and does so hyperhamiltonianly with moment map $\mu_{\mathrm{HK}}=\mu_{\mathbb{C}} \oplus \mu_{\mathbb{R}}$, where

$$
\mu_{\mathbb{R}}(z, w)=\mu(z)-\mu(w) \text { and } \mu_{\mathbb{C}}(z, w)(v)=w\left(\hat{v}_{z}\right)
$$

for $w \in T_{z}^{*} \mathbb{C}^{n}, v \in \mathfrak{g}_{\mathbb{C}}$, and $\hat{v}_{z}$ the element of $T_{z} \mathbb{C}^{n}$ induced by $v$. Consider a central regular value $\alpha \in \mathfrak{g}^{*}$ for $\mu$, and suppose that $(\alpha, 0) \in \mathfrak{g}^{*} \oplus \mathfrak{g}_{\mathbb{C}}^{*}$ is a central regular value for $\mu_{\mathrm{HK}}$. We refer to the hyperkähler reduction $M=\mathbb{H}^{n} /\|\|_{(\alpha, 0)} G$ as the hyperkähler analogue of the corresponding Kähler reduction $X=\mathbb{C}^{n} / / \alpha$. The following proposition is proven for the case where $G$ is a torus in [BD, 7.1]:

Proposition 1.2. The cotangent bundle $T^{*} X$ is isomorphic to an open subset of $M$.

Proof. Let $Y=\left\{(z, w) \in \mu_{\mathbb{C}}^{-1}(0)^{\mathrm{ss}} \mid z \in\left(\mathbb{C}^{n}\right)^{\mathrm{ss}}\right\}$, where we ask $z$ to be semistable with respect to $\alpha$ for the action of $G_{\mathbb{C}}$ on $\mathbb{C}^{n}$, so that $X \cong$ $\left(\mathbb{C}^{n}\right)^{\text {ss }} / G_{\mathbb{C}}$. Let $[z]$ denote the element of $X$ represented by $z$. The tangent 
space $T_{[z]} X$ is equal to the quotient of $T_{z} \mathbb{C}^{n}$ by the tangent space to the $G_{\mathbb{C}}$ orbit through $z$, hence

$$
\begin{aligned}
T_{[z]}^{*} X & \cong\left\{w \in T_{[z]}^{*} \mathbb{C}^{n} \mid w\left(\hat{v}_{z}\right)=0 \text { for all } v \in \mathfrak{g}_{\mathbb{C}}\right\} \\
& =\left\{w \in\left(\mathbb{C}^{n}\right)^{*} \mid \mu_{\mathbb{C}}(z, w)=0\right\} .
\end{aligned}
$$

Then

$$
T^{*} X \cong\left\{(z, w) \mid z \in\left(\mathbb{C}^{n}\right)^{\mathrm{ss}} \text { and } \mu_{\mathbb{C}}(z, w)=0\right\} / G_{\mathbb{C}}=Y / G_{\mathbb{C}}
$$

is an open subset of $M$.

Consider the action of $S^{1}$ on $\mathbb{H}^{n} \cong T^{*} \mathbb{C}^{n}$ given by "rotating the fibers" of the cotangent bundle, given explicitly by $\tau(z, w)=(z, \tau w)$. This action is hamiltonian with respect to the real symplectic structure $\omega_{\mathbb{R}}$ with moment $\operatorname{map} \Phi(z, w)=\frac{1}{2}|w|^{2}$. Because it commutes with the action of $G$, the action descends to a hamiltonian action on $M$, where we will still denote the moment map by $\Phi$. Since $S^{1}$ acts trivially on $z$, and by scalars on $w$, it does not preserve the complex symplectic form $\omega_{\mathbb{C}}(z, w)=d w \wedge d z$, and does not act $\mathbb{H}$-linearly.

Proposition 1.3. If the original moment map $\mu: \mathbb{C}^{n} \rightarrow \mathfrak{g}^{*}$ is proper, then so is $\Phi: M \rightarrow \mathbb{R}$.

Proof. We would like to show that $\Phi^{-1}[0, R]$ is compact for any $R$. Since

$$
\Phi^{-1}[0, R]=\left\{(z, w) \mid \mu_{\mathbb{R}}(z, w)=\alpha, \mu_{\mathbb{C}}(z, w)=0, \Phi(z, w) \leq R\right\} / G
$$

and $G$ is compact, it is sufficient to show that the set $\left\{(z, w) \mid \mu_{\mathbb{R}}(z, w)=\right.$ $\alpha, \Phi(z, w) \leq R\}$ is compact. Since $\mu_{\mathbb{R}}(z, w)=\mu(z)-\mu(w)$, this set is a closed subset of

$$
\mu^{-1}\left\{\alpha+\left.\mu(w)\left|\frac{1}{2}\right| w\right|^{2} \leq R\right\} \times\left\{\left.w\left|\frac{1}{2}\right| w\right|^{2} \leq R\right\},
$$

which is compact by the properness of $\mu$.

In the case where $G$ is abelian and $X$ is a nonempty toric variety, properness of $\mu$ (and therefore of $\Phi$ ) is equivalent to compactness of $X$.

\section{Hypertoric varieties.}

In this section we restrict our attention to hypertoric varieties, which are the hyperkähler analogues of toric varieties in the sense of Section 1. We begin with the full $n$-dimensional torus $T^{n}$ acting on $\mathbb{C}^{n}$, and the induced action on $\mathbb{H}^{n} \cong T^{*} \mathbb{C}^{n}$ given by $t(z, w)=\left(t z, t^{-1} w\right)$. Let $\left\{a_{i}\right\}_{1 \leq i \leq n}$ be nonzero primitive integer vectors in $\mathfrak{t}^{d} \cong \mathbb{R}^{d}$ defining a map $\beta: \mathfrak{t}^{n} \longrightarrow \mathfrak{t}^{d}$ by $\varepsilon_{i} \mapsto a_{i}$, where $\left\{\varepsilon_{i}\right\}$ is the standard basis for $\mathfrak{t}^{n} \cong \mathbb{R}^{n}$, dual to $\left\{u_{i}\right\}$. This map fits into an exact sequence

$$
0 \longrightarrow \mathfrak{t}^{k} \stackrel{\iota}{\longrightarrow} \mathfrak{t}^{n} \stackrel{\beta}{\longrightarrow} \mathfrak{t}^{d} \longrightarrow 0
$$


where $\mathfrak{t}^{k}:=\operatorname{ker}(\beta)$. Exponentiating, we get the exact sequence

$$
0 \longrightarrow T^{k} \stackrel{\iota}{\longrightarrow} T^{n} \stackrel{\beta}{\longrightarrow} T^{d} \longrightarrow 0,
$$

whereas by dualizing, we get

$$
0 \longrightarrow\left(\mathfrak{t}^{d}\right)^{*} \stackrel{\beta^{*}}{\longrightarrow}\left(\mathfrak{t}^{n}\right)^{*} \stackrel{\iota^{*}}{\longrightarrow}\left(\mathfrak{t}^{k}\right)^{*} \longrightarrow 0,
$$

where we abuse notation by using $\iota$ and $\beta$ to denote maps on the level of groups as well as on the level of algebras. Note that $T^{k}$ is connected if and only if the vectors $\left\{a_{1}, \ldots, a_{n}\right\}$ span $\mathfrak{t}^{d}$ over the integers.

Consider the restriction of the action of $T^{n}$ on $\mathbb{H}^{n}$ to the subgroup $T^{k}$. This action is hyperhamiltonian with hyperkähler moment map

$$
\begin{aligned}
& \bar{\mu}_{\mathbb{R}}(z, w)=\iota^{*}\left(\frac{1}{2} \sum_{i=1}^{n}\left(\left|z_{i}\right|^{2}-\left|w_{i}\right|^{2}\right) u_{i}\right) \quad \text { and } \\
& \bar{\mu}_{\mathbb{C}}(z, w)=\iota^{*}\left(\sum_{i=1}^{n}\left(z_{i} w_{i}\right) u_{i}\right),
\end{aligned}
$$

where $\left\{u_{i}\right\}$ is the standard basis in $\left(\mathfrak{t}^{n}\right)^{*} \cong \mathbb{R}^{n}$. In contrast with the Kähler situation, the hyperkähler moment map is surjective onto $\left(\mathfrak{t}^{n}\right)^{*} \oplus\left(\mathfrak{t}_{\mathbb{C}}^{n}\right)^{*}$.

We denote by $M$ the hyperkähler reduction of $\mathbb{H}^{n}$ by the subtorus $T^{k}$ at $(\alpha, 0) \in\left(\mathfrak{t}^{k}\right)^{*} \oplus\left(\mathfrak{t}_{\mathbb{C}}^{k}\right)^{*}$, which is the hyperkähler analogue of the Kähler toric variety $X=\mathbb{C}^{n} / / T^{k}$. Choose a lift $\widetilde{\alpha} \in\left(\mathfrak{t}^{n}\right)^{*}$ of $\alpha$ along $\iota^{*}$. Then $M$ has a natural residual action of $T^{d}$ with hyperkähler moment map $\mu_{\mathrm{HK}}=\mu_{\mathbb{R}} \oplus \mu_{\mathbb{C}}$. Note that the choice of subtorus $T^{k} \subseteq T^{n}$ is equivalent to choosing a central arrangement of cooriented hyperplanes in $\left(\mathfrak{t}^{d}\right)^{*}$, where the $i^{\text {th }}$ hyperplane is the annihilator of $a_{i} \in \mathfrak{t}^{d}$. (The coorientation comes from the fact that we know for which $x$ we have $\left\langle x, a_{i}\right\rangle>0$.) The choice of $\widetilde{\alpha}$ corresponds to an affinization $\mathcal{H}$ of this arrangement, where the $i^{\text {th }}$ hyperplane is

$$
H_{i}=\left\{x \in\left(\mathfrak{t}^{d}\right)^{*} \mid\left\langle x, a_{i}\right\rangle=\left\langle-\widetilde{\alpha}, \varepsilon_{i}\right\rangle\right\} .
$$

Changing $\widetilde{\alpha}$ by an element $c \in\left(\mathfrak{t}^{d}\right)^{*}$ has the effect of translating $\mathcal{H}$ by $c$, and adding $c$ to the residual moment map $\mu_{\mathbb{R}}$. In order to record the information about coorientations, we define the half-spaces

$$
\begin{aligned}
F_{i} & =\left\{x \in\left(\mathfrak{t}^{d}\right)^{*} \mid\left\langle x, a_{i}\right\rangle \geq\left\langle-\widetilde{\alpha}, \varepsilon_{i}\right\rangle\right\} \quad \text { and } \\
G_{i} & =\left\{x \in\left(\mathfrak{t}^{d}\right)^{*} \mid\left\langle x, a_{i}\right\rangle \leq\left\langle-\widetilde{\alpha}, \varepsilon_{i}\right\rangle\right\},
\end{aligned}
$$

which intersect in the hyperplane $H_{i}$. Our convention will be to draw pictures, as in Figure 1, in which we specify the coorientations of the hyperplanes by shading the polyhedron $\Delta=\cap_{i=1}^{n} F_{i}$ (which works as long as $\Delta \neq \emptyset)$. Note that the Kähler variety $X$ is precisely the Kähler toric variety determined by $\Delta$. 


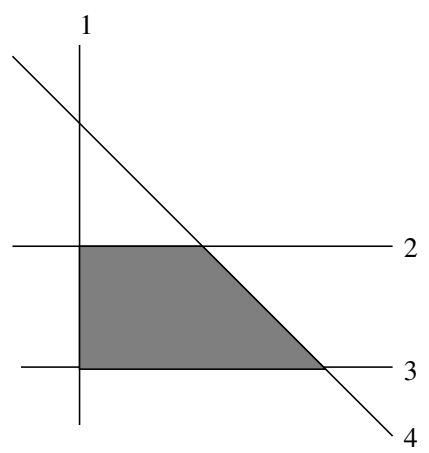

Figure 1. A hypertoric variety of real dimension 8 obtained by reducing $\mathbb{H}^{4}$ by $T^{2}$.

The variety $M$ is an orbifold if and only if $\mathcal{H}$ is simple, i.e., if and only if every subset of $m$ hyperplanes intersect in codimension $m$ [BD, 3.2]. Furthermore, $M$ is smooth if and only if whenever some subset of $d$ hyperplanes $\left\{H_{i}\right\}$ has nonempty intersection, the corresponding vectors $\left\{a_{i}\right\}$ form a $\mathbb{Z}$ basis for $\mathbb{Z}^{d} \subseteq \mathfrak{t}^{d}$. In this case we will refer to the arrangement itself as smooth. We will always assume that $\mathcal{H}$ is simple, and at times we will also assume that it is smooth.

The hyperplanes $\left\{H_{i}\right\}$ divide $\left(\mathfrak{t}^{d}\right)^{*} \cong \mathbb{R}^{d}$ into a finite family of closed, convex polyhedra

$$
\Delta_{A}=\left(\cap_{i \in A} F_{i}\right) \cap\left(\cap_{i \notin A} G_{i}\right),
$$

indexed by subsets $A \subseteq\{1, \ldots, n\}$. Consider the subset

$$
I=\left\{A \subseteq\{1, \ldots, n\} \mid \Delta_{A} \text { bounded }\right\}
$$

of the power set of $\{1, \ldots, n\}$. For each $A \subseteq\{1, \ldots, n\}$, let

$$
M_{A}=\mu_{\mathbb{R}}^{-1}\left(\Delta_{A}\right) \cap \mu_{\mathbb{C}}^{-1}(0) .
$$

The Kähler submanifold $\left(M_{A},\left.\omega_{\mathbb{R}}\right|_{M_{A}}\right)$ of $\left(M, \omega_{\mathbb{R}}\right)$ is $d$-dimensional and invariant under the action of $T^{d}$, and is therefore $T^{d}$-equivariantly isomorphic to the Kähler toric variety determined by $\Delta_{A}[\mathbf{B D}, 6.5]$. We define the core $C$ and extended core $D$ of a hypertoric variety by setting

$$
C=\cup_{A \in I} M_{A} \text { and } D=\cup_{A} M_{A}=\mu_{\mathbb{C}}^{-1}(0)=\left\{[z, w] \mid z_{i} w_{i}=0 \text { for all } i\right\},
$$

where $[z, w]$ denotes the $T^{k}$-equivalence class in $M$ of the element $(z, w) \in$ $\bar{\mu}_{\mathrm{HK}}^{-1}(\alpha, 0)$. Bielawski and Dancer $[\mathrm{BD}]$ show that $C$ and $D$ are each $T^{d_{-}}$ equivariant deformation retracts of $M$. See Corollary 3.6 for a Morse theoretic proof.

We take a minute to discuss the differences between the combinatorial data determining a toric variety $X=\mathbb{C}^{n} / / T^{k}$ and its hypertoric analogue $M=\mathbb{H}^{n} /\|\|_{(\alpha, 0)} T^{k}$. Each is determined by $\mathcal{H}$, a simple, cooriented, affine 
arrangement of $n$ hyperplanes in $\left(\mathfrak{t}^{d}\right)^{*}$, defined up to simultaneous translation. The toric variety $X$ is in fact determined by less information than this; it depends only on the polyhedron $\Delta=\cap_{i=1}^{n} F_{i}$. Thus if the last hyperplane $H_{n}$ has the property that $\cap_{i=1}^{n-1} F_{i} \subseteq F_{n}$, then this hyperplane is superfluous to $X$. This is not the case for $M$, which means that it is slightly misleading to call $M$ the hyperkähler analogue of $X$; more precisely, it is the hyperkähler analogue of a given presentation of $X$ as a Kähler reduction of $\mathbb{C}^{n}$. On the other hand, the $T^{d}$-equivariant diffeomorphism type of $M$ also does not depend on all of the information of $\mathcal{H}$, as evidenced by the two following results:

Lemma 2.1. The hypertoric varieties $M_{\alpha}=\mathbb{H}^{n} /\|\|_{(\alpha, 0)} T^{k}$ and $M_{\alpha^{\prime}}=$ $\mathbb{H}^{n} / \|\left.\right|_{\left(\alpha^{\prime}, 0\right)} T^{k}$ are $T^{d}$-equivariantly diffeomorphic, and their cohomology rings can be naturally identified.

Lemma 2.2. The hypertoric variety $M$ does not depend on the coorientations of the hyperplanes $\left\{H_{i}\right\}$.

This means that, unlike that of $X$, the $T^{d}$-equivariant diffeomorphism type of $M$ depends only on the unoriented central arrangement underlying $\mathcal{H}$. A weaker version of Lemma 2.1, involving the (nonequivariant) homeomorphism type of $M$, appears in [BD].

Proof of 2.1. The set of nonregular values for $\bar{\mu}_{\mathrm{HK}}$ has codimension 3 inside of $\left(\mathfrak{t}^{d}\right)^{*} \oplus\left(\mathfrak{t}_{\mathbb{C}}^{d}\right)^{*}$. This tells us that the set of regular values is simply connected, and we can choose a path connecting any two regular values $(\alpha, 0)$ and $\left(\alpha^{\prime}, 0\right)$, unique up to homotopy.

Since the moment map $\bar{\mu}_{\mathrm{HK}}$ is not proper, we must take some care in showing that two fibers are diffeomorphic. To this end, we note that the norm-square function $\psi(z, w)=\|z\|^{2}+\|w\|^{2}$ is $T^{n}$-invariant and proper on $\mathbb{H}^{n}$. Let $\mathbb{H}_{\text {reg }}^{n}$ denote the open submanifold of $\mathbb{H}^{n}$ consisting of the preimages of the regular values of $\bar{\mu}_{\mathrm{HK}}$. By a direct computation, it is easy to see that the kernels of $d \psi$ and $d\left(\bar{\mu}_{\mathrm{HK}}\right)$ intersect transversely at any point $p \in \mathbb{H}_{\text {reg }}^{n}$. Using the standard $T^{n}$-invariant metric on $\mathbb{H}^{n}$, we define an Ehresmann connection on $\mathbb{H}_{\text {reg }}^{n}$ with respect to $\bar{\mu}_{\mathrm{HK}}$ such that the horizontal subspaces are contained in the kernel of $d \psi$.

This connection allows us to lift a path connecting the two regular values to a horizontal vector field on its preimage in $\mathbb{H}_{\text {reg. Since the horizontal }}^{n}$ subspaces are tangent to the kernel of $d \psi$, the flow preserves level sets of $\psi$. Note that the function

$$
\bar{\mu}_{\mathrm{HK}} \oplus \psi: \mathbb{H}^{n} \rightarrow\left(\mathfrak{t}^{d}\right)^{*} \oplus\left(\mathfrak{t}_{\mathbb{C}}^{d}\right)^{*} \oplus \mathbb{R}
$$

is proper. By a theorem of Ehresmann [BJ, 8.12], the properness of this map implies that the flow of this vector field exists for all time, and identifies the inverse image of $(\alpha, 0)$ with that of $\left(\alpha^{\prime}, 0\right)$. Since the metric, $\psi$, and 
$\bar{\mu}_{\mathrm{HK}}$ are all $T^{n}$-invariant, the Ehresmann connection is also $T^{n}$-invariant, therefore the diffeomorphism identifying the fibers is $T^{n}$-equivariant, making the reduced spaces are $T^{d}$-equivariantly diffeomorphic.

Proof of 2.2. It suffices to consider the case when we change the orientation of a single hyperplane within the arrangement. Changing the coorientation of a hyperplane $H_{l}$ is equivalent to defining a new map $\beta^{\prime}: \mathfrak{t}^{n} \rightarrow \mathfrak{t}^{d}$, with $\beta^{\prime}\left(\varepsilon_{i}\right)=a_{i}$ for $i \neq l$, and $-a_{i}$ for $i=l$. This map exponentiates to a map $\beta^{\prime}: T^{n} \rightarrow T^{d}$, and we want to show that the hyperkähler variety obtained by reducing $\mathbb{H}^{n}$ by the torus $\operatorname{ker}\left(\beta^{\prime}\right)$ is isomorphic to $M$, which is obtained by reducing $\mathbb{H}^{n}$ by the torus $T^{k}=\operatorname{ker}(\beta)$. To see this, note that $\operatorname{ker}\left(\beta^{\prime}\right)$ and $\operatorname{ker}(\beta)$ are conjugate inside of $\mathrm{M}(n, \mathbb{H})$ by the element $(1, \ldots, 1, j, 1, \ldots, 1) \in$ $\mathrm{M}(1, \mathbb{H})^{n} \subseteq \mathrm{M}(n, \mathbb{H})$, where the $j$ appears in the $l^{\text {th }}$ slot.

Example 2.3. The three cooriented arrangements of Figure 2 all specify the same hyperkähler variety $M$ up to equivariant diffeomorphism. The first has $X \cong \mathbb{F}_{1}$ (the first Hirzebruch surface) and the second and the third have $X \cong \mathbb{C} P^{2}$. Note that if we flipped the coorientation of $H_{3}$ in Figure 2(a) or $2(\mathrm{c})$, then we would get a noncompact $X \cong \widetilde{\mathbb{C}}^{2}$, the blow-up of $\mathbb{C}^{2}$ at a point. If we flipped the coorientation of $H_{3}$ in Figure 2(b), then $X$ would be empty. We make no assumptions about $X$ in this section.

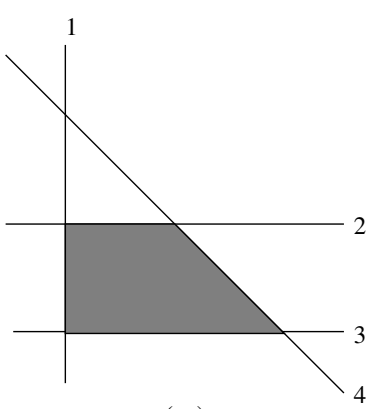

( a )

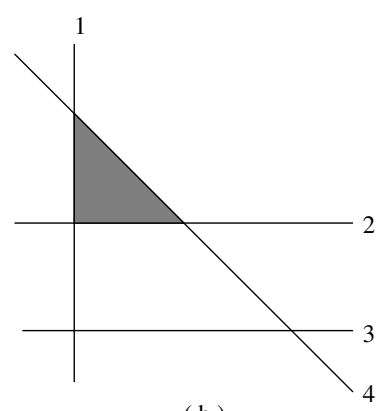

( b )

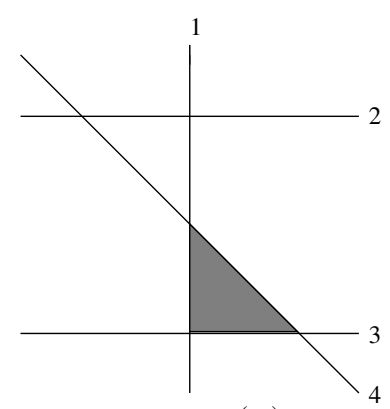

(c)

Figure 2. Three arrangements related by flipping coorientations and translating hyperplanes.

The purpose of this paper is to study not just the topology of $M$, but the topology of $M$ along with the natural hamiltonian $S^{1}$ action defined in Section 1. In order to define this $S^{1}$ action, it is necessary that we reduce at a regular value of the form $(\alpha, 0) \in\left(\mathfrak{t}^{d}\right)^{*} \oplus\left(\mathfrak{t}_{\mathbb{C}}^{d}\right)^{*}$, and although the set of regular values of $\bar{\mu}_{\mathrm{HK}}$ is simply connected, the set of regular values of the form $(\alpha, 0)$ is not even connected. Furthermore, left multiplication by the diagonal matrix $(1, \ldots, 1, j, 1, \ldots, 1) \in \mathrm{U}(n, \mathbb{H})$ is not an $S^{1}$-equivariant automorphism of $\mathbb{H}^{n}$, therefore the geometric structure of $M$ along with 
a circle action may depend nontrivially both on the affine structure and the coorientations of the arrangement $\mathcal{H}$. Indeed it must, because we can recover $X$ from $M$ by taking the minimum $\Phi^{-1}(0)$ of the $S^{1}$ moment map $\Phi: M \rightarrow \mathbb{R}$. In this sense, the structure of a hypertoric variety $M$ along with a circle action is the universal geometric object from which both $M$ and $X$ can be recovered.

\section{Gradient flow on the core.}

Although $S^{1}$ does not act on $M$ as a subtorus of $T^{d}$, we show below that when restricted to any single component $M_{A}$ of the extended core, $S^{1}$ does act as a subtorus of $T^{d}$, with the subtorus depending combinatorially on $A$. This will allow us to give a combinatorial analysis of the gradient flow of $\Phi$ on the extended core.

Lemma 3.1. Let $x$ be an element of $\left(\mathfrak{t}^{d}\right)^{*}$, and consider a point $[z, w] \in$ $\mu_{\mathbb{R}}^{-1}(x) \cap D$. We have $x \in F_{i}$ if and only if $w_{i}=0$, and $x \in G_{i}$ if and only if $z_{i}=0$.

Proof. The fact that $[z, w] \in D$ tells us that $z_{i} w_{i}=0$. Then

$$
\begin{aligned}
x \in F_{i} & \Leftrightarrow\left\langle-\widetilde{\alpha}, \varepsilon_{i}\right\rangle \leq\left\langle\mu_{\mathbb{R}}[z, w], a_{i}\right\rangle=\left\langle\overline{\mu_{\mathbb{R}}}(z, w)-\widetilde{\alpha}, \varepsilon_{i}\right\rangle \\
& \Leftrightarrow \frac{1}{2}\left|z_{i}\right|^{2}-\frac{1}{2}\left|w_{i}\right|^{2}=\left\langle\overline{\mu_{\mathbb{R}}}(z, w), \varepsilon_{i}\right\rangle \geq 0 .
\end{aligned}
$$

Since $z_{i} w_{i}=0$, this is equivalent to the condition $w_{i}=0$. The second half of the lemma follows similarly.

On the suborbifold $M_{A} \subseteq D \subseteq M$ we have $z_{i}=0$ for all $i \in A$ and $w_{i}=0$ for all $i \notin A$, therefore for $\tau \in S^{1}$ and $[z, w] \in M_{A}$,

$$
\begin{array}{r}
\tau[z, w]=[z, \tau w]=\left[\tau_{1} z_{1}, \ldots, \tau_{n} z_{n}, \tau_{1}^{-1} w_{1}, \ldots, \tau_{n}^{-1} w_{n}\right], \\
\text { where } \tau_{i}= \begin{cases}\tau^{-1} & \text { if } i \in A, \\
1 & \text { if } i \notin A .\end{cases}
\end{array}
$$

In other words, the $S^{1}$ action on $M_{A}$ is given by the one dimensional subtorus $\left(\tau_{1}, \ldots, \tau_{n}\right)$ of the original torus $T^{n}$, hence the moment map $\left.\Phi\right|_{M_{A}}$ is given (up to an additive constant) by

$$
\Phi[z, w]=\left\langle\mu_{\mathbb{R}}[z, w], \sum_{i \in A} a_{i}\right\rangle .
$$

This formula allows us to compute the fixed point sets of the $S^{1}$ action. Since $S^{1}$ acts freely on $\left(\mathfrak{t}_{\mathbb{C}}^{d}\right)^{*} \backslash\{0\}$ and $\mu_{\mathbb{C}}: M \rightarrow\left(\mathfrak{t}_{\mathbb{C}}^{d}\right)^{*}$ is $S^{1}$-equivariant, we must have $M^{S^{1}} \subseteq \mu_{\mathbb{C}}^{-1}(0)=D$. For any subset $B \subseteq\{1, \ldots, n\}$, let $M_{A}^{B}$ be the toric subvariety of $M_{A}$ defined by the conditions $z_{i}=w_{i}=0$ 
for all $i \in B$. Geometrically, $M_{A}^{B}$ is defined by the (possibly empty) face $\cap_{i \in B} H_{i} \cap \Delta_{A}$ of the polyhedron $\Delta_{A}$.

Proposition 3.2. The fixed point set of the action of $S^{1}$ on $M_{A}$ is the union of those toric subvarieties $M_{A}^{B}$ such that $\sum_{i \in A} a_{i} \in \mathfrak{t}_{B}^{d}:=\operatorname{Span}_{j \in B} a_{j}$.

Proof. The moment map $\left.\Phi\right|_{M_{A}^{B}}$ will be constant if and only if $\sum_{i \in A} a_{i}$ is perpendicular to $\operatorname{ker}\left(\left(\mathfrak{t}^{d}\right)^{*} \rightarrow\left(\mathfrak{t}_{B}^{d}\right)^{*}\right)$, i.e., if $\sum_{i \in A} a_{i}$ lies in the kernel of the projection $\mathfrak{t}^{d} \rightarrow \mathfrak{t}^{d} / \mathfrak{t}_{B}^{d}$.

Corollary 3.3. Every vertex $v \in\left(\mathfrak{t}^{d}\right)^{*}$ of the polyhedral complex defined by $\mathcal{H}$ is the image of an $S^{1}$-fixed point in $M$. Every component of $M^{S^{1}}$ has dimension less than or equal to $d$, and the only component of dimension $d$ is $M_{\emptyset}=X=\Phi^{-1}(0)$.

For any point $p \in M^{S^{1}}$, the stable orbifold $S(p)$ at $p$ is defined to be the set of $x \in M$ such that $x$ approaches $p$ when flowing along the vector field $-\operatorname{grad}(\Phi)$, and the unstable orbifold $U(p)$ at $p$ is defined to be the stable orbifold with respect to the function $-\Phi$. For any suborbifold $Y \subseteq M^{S^{1}}$, the unstable orbifold $U(Y)$ at $Y$ is defined to be the union of $U(y)$ for all $y \in Y$. In general, for $y \in Y$, we have the identity $\operatorname{dim}_{\mathbb{R}} U(Y)+\operatorname{dim}_{\mathbb{R}} S(y)=4 d$.

Let $Y \subseteq M^{S^{1}}$ be a component of the fixed point set of $M$. Let $v \in\left(\mathfrak{t}^{d}\right)^{*}$ be a vertex in the polehedron $\mu_{\mathbb{R}}(Y)$, and let $y$ be the unique preimage of $v$ in $Y$.

Proposition 3.4. The unstable orbifold $U(Y)$ is a complex suborbifold of complex dimension at most $d$, contained in the core $C \subseteq M$. If $\mathcal{H}$ is smooth at $y$, then $\operatorname{dim}_{\mathbb{C}} U(Y)=d$, and the closure of $U(Y)$ is an irreducible component of $C$.

Proof. For simplicity, we will assume that $v=\cap_{j=1}^{d} H_{j}$. For all $l \in\{1, \ldots, d\}$, let $b_{l} \in \mathfrak{t}_{\mathbb{Z}}^{d}$ be the smallest integer vector such that $\left\langle a_{j}, b_{l}\right\rangle=0$ for $j \neq l$ and $\left\langle a_{l}, b_{l}\right\rangle>0$. Geometrically, $b_{l}$ is the primitive integer vector on the line $\cap_{j \neq l} H_{j}$ pointing in the direction of $\Delta$. Note that $M$ is smooth at the $T^{d}$-fixed point above $v$ if and only if $\left\langle a_{l}, b_{l}\right\rangle=1$ for all $l \in\{1, \ldots, d\}$. Let $R_{l} \subseteq\left(\mathfrak{t}^{d}\right)^{*}$ be the ray eminating from $v$ in the direction of $b_{l}$, and ending before it hits another vertex. Let $Q_{l}$ be the analogous ray in the opposite direction.

Let $\Delta_{A}$ be a region (not necessarily bounded) of the polyhedral complex defined by $\mathcal{H}$ adjacent to $R_{l}$. The preimage $\mu_{\mathbb{R}}^{-1}\left(R_{l}\right) \cap D$ of $R_{l}$ in $D$ is a complex line, and it is contained in the unstable orbifold at $U(Y)$ if and only if $\left\langle b_{l}, \sum_{i \in A} a_{i}\right\rangle \geq 0$. If $\left\langle b_{l}, \sum_{i \in A} a_{i}\right\rangle<0$, it is contained in the stable orbifold $S(y)$. The preimage $\mu_{\mathbb{R}}^{-1}\left(Q_{l}\right) \cap D$ of $Q_{l}$ in $D$ is also a complex line, contained in the unstable orbifold $U(Y)$ if and only if $\left\langle-b_{l}, a_{l}+\sum_{i \in A} a_{i}\right\rangle \geq 0$, and otherwise in $S(y)$. Since $\left\langle b_{l}, \sum_{i \in A} a_{i}\right\rangle+\left\langle-b_{l}, a_{l}+\sum_{i \in A} a_{i}\right\rangle=-\left\langle a_{l}, b_{l}\right\rangle<0$, 
at most one of these two directions can be unstable. In the smooth case, $\left\langle a_{l}, b_{l}\right\rangle=1$ for all $l$, and exactly one of the two directions is unstable.

Consider the polytope $\Delta_{v}$ incident to $v$ and characterized by the property that its edges at the vertex $v$ are exactly the unstable directions. The toric variety $X_{\Delta_{v}} \subseteq D$ is contained in the closure of $U(Y)$, and a dimension count tells us that this containment is an equality. In the smooth case, $\Delta_{v}$ is $d$-dimensional, and $X_{\Delta_{v}}$ is a component of the core.

Note that, even in the smooth case, it is not necessarily the case that the $R_{l}$ direction is stable and the $Q_{l}$ direction is unstable. See, for example, the vertex $v=H_{1} \cap H_{2}$ in Figure 2(c).

Corollary 3.5. There is a natural injection from the set of bounded regions $\left\{\Delta_{A} \mid A \in I\right\}$ to the set of connected components of $M^{S^{1}}$. If $\mathcal{H}$ is smooth, this map is a bijection.

Proof. To each $A \in I$, we associate the fixed subvariety $M_{A}^{B}$ corresponding to the face of $\Delta_{A}$ on which the linear functional $\sum_{i \in A} a_{i}$ is minimized, so that $M_{A}$ is the closure of $U\left(M_{A}^{B}\right)$. If $\mathcal{H}$ is smooth, then every connected component of the fixed point set will have a component of the core as its closed unstable orbifold.

Corollary 3.6. The core of $M$ is equal to the union of the unstable orbifolds of the connected components of $M^{S^{1}}$, hence $C$ is a $T^{d} \times S^{1}$-equivariant deformation retract of $M$.

Example 3.7. In Figure 3, representing a reduction of $\mathbb{H}^{5}$ by $T^{3}$, we choose a metric on $\left(\mathfrak{t}^{2}\right)^{*}$ in order to draw the linear functional $\sum_{i \in A} a_{i}$ as a vector in each region $\Delta_{A}$. We see that $M^{S^{1}}$ has three components, one of them $X \cong \mathbb{F}_{1}$, one of them a projective line, with another $\mathbb{F}_{1}$ as its unstable manifold, and one of them a point, with a $\mathbb{C} P^{2}$ as its unstable manifold.

Example 3.8. The hypertoric variety represented by Figure 4 has a fixed point set with four connected components (three points and a $\mathbb{C} P^{2}$ ), but only three components in its core. This phenomenon can be blamed on the orbifold point represented by the intersection of $H_{3}$ and $H_{4}$, which has a one-dimensional unstable orbifold.

\section{Equivariant cohomology.}

In this section we extend Konno's computations of the ordinary and $T^{d_{-}}$ equivariant cohomologies of $M$ to the $S^{1}$-equivariant setting. We follow Konno's approach of restricting to the smooth case to simplify arguments involving line bundles on $M$. Hausel and Sturmfels, however, prove theorems analogous to 4.1 and 4.3 with rational coefficients in the orbifold case, and Theorems 4.4 and 4.5 extend to this setting as well (see Remark 4.12). 


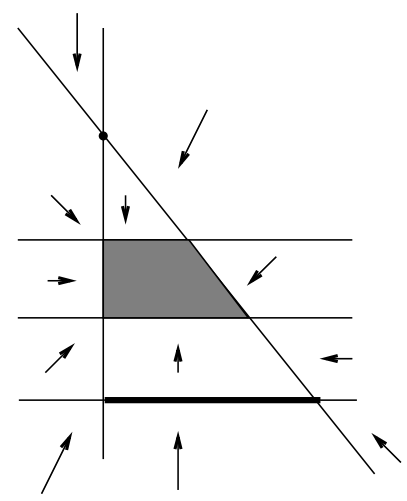

Figure 3. The gradient flow of $\Phi: M \rightarrow \mathbb{R}$.

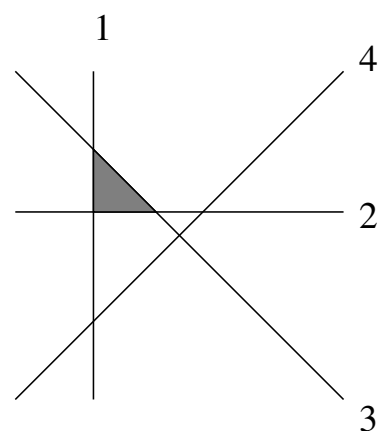

Figure 4. A singular example.

Theorem 4.1 ([K2]). The $T^{d}$-equivariant cohomology ring of a smooth hypertoric variety $M$ is given by

$$
H_{T^{d}}^{*}(M)=\mathbb{Z}\left[u_{1}, \ldots, u_{n}\right] /\left\langle\prod_{i \in S} u_{i} \mid \bigcap_{i \in S} H_{i}=\emptyset\right\rangle .
$$

Remark 4.2. This is precisely the Stanley-Reisner ring of the unoriented matroid determined by the arrangement $\mathcal{H}[\mathbf{H S}]$.

Just as the cohomology of a toric variety is obtained from the equivariant cohomology by introducing linear relations that generate $\operatorname{ker} \iota^{*}=(\operatorname{ker} \beta)^{\perp}$, the same is true for hypertoric varieties:

Theorem 4.3 ([K1]). The ordinary cohomology ring of a smooth hypertoric variety $M$ is given by

$$
H^{*}(M)=H_{T^{d}}^{*}(M) /\left\langle\Sigma a_{i} u_{i} \in \operatorname{ker} \iota^{*}\right\rangle .
$$

The rest of this section will be devoted to the proof of the following two theorems: 
Theorem 4.4. Let $M$ be the hypertoric variety corresponding to a smooth, cooriented arrangement $\mathcal{H}$. Given any minimal set $S \subseteq\{1, \ldots, n\}$ such that $\cap_{i \in S} H_{i}=\emptyset$, let $S=S_{1} \sqcup S_{2}$ be the unique splitting of $S$ such that $\left(\cap_{i \in S_{1}} G_{i}\right) \cap\left(\cap_{j \in S_{2}} F_{j}\right)=\emptyset($ see $(1))$. Then the $T^{d} \times S^{1}$-equivariant cohomology of $M$ is given by

$$
H_{T^{d} \times S^{1}}^{*}(M) \cong \mathbb{Z}\left[u_{1}, \ldots, u_{n}, x\right] /\left\langle\prod_{i \in S_{1}} u_{i} \times \prod_{j \in S_{2}}\left(x-u_{j}\right) \mid \bigcap_{i \in S} H_{i}=\emptyset\right\rangle .
$$

Theorem 4.5. In the notation of Theorem 4.4, the $S^{1}$-equivariant cohomology ring of $M$ is given by

$$
H_{S^{1}}^{*}(M) \cong H_{T^{d} \times S^{1}}^{*}(M) /\left\langle\Sigma a_{i} u_{i} \in \operatorname{ker} \iota^{*}\right\rangle .
$$

Remark 4.6. Konno observes that the quotient map from the abstract polynomial ring $\mathbb{Z}\left[u_{1}, \ldots, u_{n}\right] \rightarrow H_{T^{d}}^{*}(M)$ is precisely the $T^{d}$-equivariant Kirwan map

$$
\kappa_{T^{d}}: H_{T^{n}}^{*}\left(T^{*} \mathbb{C}^{n}\right) \rightarrow H_{T^{d}}^{*}(M)
$$

which is induced by the inclusion $\mu^{-1}(\alpha, 0) \hookrightarrow T^{*} \mathbb{C}^{n}$. Likewise, the map from $\mathbb{Z}\left[u_{1}, \ldots, u_{n}\right] / \operatorname{ker} \iota^{*}$ to $H^{*}(M)$ is the ordinary Kirwan map

$$
\kappa: H_{T^{k}}^{*}\left(T^{*} \mathbb{C}^{n}\right) \rightarrow H^{*}(M) .
$$

The analogous maps for Kähler reductions are known to always be surjective [Ki, 5.4], but the hyperkähler case remains open. Thus Theorems 4.1 and 4.3 can be interpreted as saying that the Kirwan maps for hypertoric varieties are surjective, and computing the kernel. Likewise, Theorems 4.4 and 4.5 assert that the $S^{1}$-equivariant Kirwan maps

$$
\kappa_{T^{d} \times S^{1}}: H_{T^{n} \times S^{1}}^{*}\left(T^{*} \mathbb{C}^{n}\right) \rightarrow H_{T^{d} \times S^{1}}^{*}(M)
$$

and

$$
\kappa_{S^{1}}: H_{T^{k} \times S^{1}}^{*}\left(T^{*} \mathbb{C}^{n}\right) \rightarrow H_{S^{1}}^{*}(M)
$$

are surjective, and provide computations of their kernels.

In order to apply Konno's results, we will make use of the principle of equivariant formality, proven for compact manifolds in $[\mathbf{K i}]$, which we adapt to our situation in Proposition 4.7. For the sake of simplicity, we will restrict our attention to the case where $X$ is compact and nonempty. This condition will be necessary for the application of Proposition 4.7 and the proof of Theorem 5.1, both of which require a proper Morse function, which we get from Proposition 1.3. We note, however, that both Proposition 4.7 and Theorem 5.1 can be extended to the case of a general hypertoric variety by a Mayer-Vietoris argument, using the fact that the core $C \subseteq M$ is a compact $T^{d} \times S^{1}$-equivariant deformation retract. We present the slightly less general Morse theoretic proofs only because we find them more pleasant. 
Proposition 4.7. Let $M$ be a symplectic orbifold, possibly noncompact but of finite topological type. Suppose that $M$ admits a hamiltonian action of a torus $T \times S^{1}$, and that the $S^{1}$-component $\Phi: M \rightarrow \mathbb{R}$ of the moment map is proper and bounded below. Then $H_{T \times S^{1}}^{*}(M)$ is a free module over $H_{S^{1}}^{*}(\mathrm{pt})$.

Proof. Because $\Phi$ is a moment map, it is a Morse-Bott function such that all of the critical suborbifold and their normal bundles carry almost complex structures. Thus we get a Morse-Bott stratification of $M$ into evendimensional $T$-invariant suborbifolds. This tells us, as in $[\mathbf{K i}, 5.8]$, that the spectral sequence associated to the fibration $M \hookrightarrow E G \times{ }_{G} M \rightarrow B G$ collapses, and we get the desired result.

Consider the following commuting square of maps, where $\phi$ and $\psi$ are each given by setting $x$ to zero:

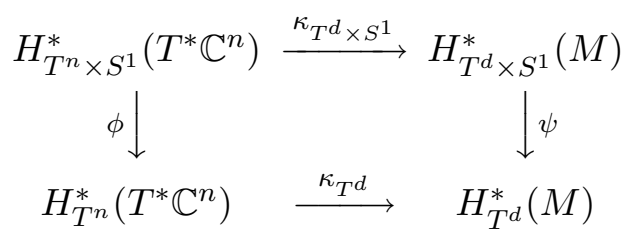

Proposition 4.7 has the following consequence:

Corollary 4.8. Let $\mathcal{I} \subseteq \operatorname{ker} \kappa_{T^{d} \times S^{1}}$ be an ideal with $\phi(\mathcal{I})=\operatorname{ker} \kappa_{T^{d}}$. Then $\mathcal{I}=\operatorname{ker} \kappa_{T^{d} \times S^{1}}$.

Proof. Suppose that $a \in \operatorname{ker} \kappa_{T^{d} \times S^{1}} \backslash \mathcal{I}$ is a homogeneous class of minimal degree, and choose $b \in \mathcal{I}$ such that $\phi(a-b)=0$. Then $a-b=c x$ for some $c \in H_{T^{n} \times S^{1}}^{*}\left(T^{*} \mathbb{C}^{n}\right)$. By Proposition 4.7, cx $\in \operatorname{ker} \kappa_{T^{d} \times S^{1}} \Rightarrow c \in \operatorname{ker} \kappa_{T^{d} \times S^{1}}$, hence $c \in \operatorname{ker} \kappa_{T^{d} \times S^{1}} \backslash \mathcal{I}$ is a class of lower degree than $a$.

Lemma 4.9. The equivariant Kirwan map $\kappa_{T^{d} \times S^{1}}$ is surjective.

Proof. Suppose that $\gamma \in H_{T^{d} \times S^{1}}^{*}(M)$ is a homogeneous class of minimal degree that is not in the image of $\kappa_{T^{d} \times S^{1}}$. By Theorem $4.1 \kappa_{T^{d}}$ is surjective, hence we may choose a class $\eta \in \phi^{-1} \kappa_{T^{d}}^{-1} \psi(\gamma)$. Then $\kappa_{T^{d} \times S^{1}}(\eta)-\gamma=x \delta$ for some $\delta \in H_{T^{d} \times S^{1}}^{*}(M)$, and therefore $\delta$ is a class of lower degree that is not in the image of $\kappa_{T^{d} \times S^{1}}$.

Proof of 4.4. For any element $h \in H_{T^{n} \times S^{1}}^{2}\left(\mathbb{H}^{n} ; \mathbb{Z}\right)$, let $\widetilde{L}_{h}=\mathbb{H}^{n} \times \mathbb{C}_{h}$ be the $T^{n} \times S^{1}$-equivariant line bundle on $\mathbb{H}^{n}$ with equivariant Euler class $h$. This gives $\widetilde{L}_{h}$, as well as its dual $\widetilde{L}_{h}^{*}$, the structure of a $T^{n} \times S^{1}$-equivariant bundle. Let

$$
L_{h}=\left.\widetilde{L}_{h}\right|_{\bar{\mu}_{\mathbb{C}}^{-1}(0)^{\mathrm{ss}}} / T_{\mathbb{C}}^{k}
$$

be the quotient $T^{d} \times S^{1}$-equivariant line bundle on $M$. Let $\left\{u_{i}\right\}$ be the standard basis of $\left(\mathfrak{t}_{\mathbb{Z}}^{n}\right)^{*}$. Identifying $H_{T^{n} \times S^{1}}^{2}\left(\mathbb{H}^{n} ; \mathbb{Z}\right)$ with $\left(\mathfrak{t}_{\mathbb{Z}}^{n}\right)^{*} \oplus \mathbb{Z} x$, we 
will use $\widetilde{L}_{i}$ to denote the bundle $\widetilde{L}_{u_{i} \oplus 0}$, and $\widetilde{K}$ to denote the bundle $\widetilde{L}_{0 \oplus x}$, with quotients $L_{i}$ and $K$. Since the $T^{d} \times S^{1}$-equivariant Euler class $e\left(L_{i}\right)$ is the image of $u_{i} \oplus 0$ under the hyperkähler Kirwan map $H_{T^{n} \times S^{1}}^{*}\left(\mathbb{H}^{n}\right) \rightarrow$ $H_{T^{d} \times S^{1}}^{*}(M)$, we will abuse notation and denote it by $u_{i}$. Similarly, we will denote $e(K)$ by $x$. Corollary 4.9 tells us that $H_{T^{d} \times S^{1}}^{*}(M)$ is generated by $u_{1}, \ldots, u_{n}, x$.

Consider the $T^{n} \times S^{1}$-equivariant section $\widetilde{s}_{i}$ of $\widetilde{L}_{i}$ given by the function $\tilde{s}_{i}(z, w)=z_{i}$. This descends to a $T^{d} \times S^{1}$-equivariant section $s_{i}$ of $L_{i}$ with zero-set

$$
Z_{i}:=\left\{[z, w] \in M \mid z_{i}=0\right\} .
$$

Similarly, the function $\widetilde{t}_{i}(z, w)=w_{i}$ defines a $T^{d} \times S^{1}$-equivariant section of $L_{i}^{*} \otimes K$ with zero set

$$
W_{i}:=\left\{[z, w] \in M \mid w_{i}=0\right\} .
$$

Thus the divisor $Z_{i}$ represents the cohomology class $u_{i}$, and $W_{i}$ represents $x-u_{i}$. Note, by the proof of Lemma 3.1, that $\mu_{\mathbb{R}}\left(Z_{i}\right)=G_{i}$ and $\mu_{\mathbb{R}}\left(W_{i}\right)=F_{i}$ for all $1 \leq i \leq n$.

Let $S=S_{1} \sqcup S_{2}$ be a subset of $\{1, \ldots, n\}$ such that

$$
\left(\cap_{i \in S_{1}} G_{i}\right) \cap\left(\cap_{j \in S_{2}} F_{j}\right)=\emptyset
$$

and hence

$$
\left(\cap_{i \in S_{1}} Z_{i}\right) \cap\left(\cap_{j \in S_{2}} W_{j}\right) \subseteq \mu_{\mathbb{R}}^{-1}\left(\left(\cap_{i \in S_{1}} G_{i}\right) \cap\left(\cap_{j \in S_{2}} F_{j}\right)\right)=\emptyset .
$$

Now consider the vector bundle $E_{S}=\left(\oplus_{i \in S_{1}} L_{i}\right) \oplus\left(\oplus_{j \in S_{2}} L_{j}^{*} \otimes K\right)$ with equivariant Euler class

$$
e\left(E_{S}\right)=\prod_{i \in S_{1}} u_{i} \times \prod_{j \in S_{2}}\left(x-u_{j}\right) .
$$

The section $\left(\oplus_{i \in S_{1}} s_{i}\right) \oplus\left(\oplus_{i \in S_{2}} t_{i}\right)$ is a nonvanishing equivariant global section of $E_{S}$, hence for any such $S, e\left(E_{S}\right)$ is trivial in $H_{T^{d} \times S^{1}}^{*}(M)$.

The fact that $u_{1}, \ldots, u_{n}, x$ generate $H_{T^{d} \times S^{1}}^{*}(M)$ is proven in Lemma 4.9, and the fact that we have found all of the relations follows from Theorem 4.1 and Corollary 4.8.

Proof of 4.5. The proof of this theorem is identical to the proof of Theorem 4.4, making use of Theorem 4.3 rather than Theorem 4.1.

How sensitive are the invariants $H_{T^{d} \times S^{1}}^{*}(M)$ and $H_{S^{1}}^{*}(M)$ ? We can recover $H_{T^{d}}^{*}(M)$ and $H^{*}(M)$ by setting $x$ to zero, hence they are at least as fine as the ordinary or $T^{d}$-equivariant cohomology rings. The ring $H_{T^{d} \times S^{1}}^{*}(M)$ does not depend on coorientations, for if $M^{\prime}$ is related to $M$ by flipping the coorientation of the $l^{\text {th }}$ hyperplane $H_{k}$, then the map taking $u_{i}$ to $u_{i}$ for $i \neq l$ 
and $u_{l}$ to $x-u_{l}$ is an isomorphism between $H_{T^{d} \times S^{1}}^{*}(M)$ and $H_{T^{d} \times S^{1}}^{*}\left(M^{\prime}\right)$. It is, however, dependent on the affine structure of the arrangement $\mathcal{H}$.

Example 4.10. We compute the equivariant cohomology ring $H_{T^{d} \times S^{1}}^{*}(M)$ for the hypertoric varieties $M_{a}, M_{b}$, and $M_{c}$ defined by the arrangements in Figure 2(a), (b), and (c), respectively.

$$
\begin{aligned}
& H_{T^{d} \times S^{1}}^{*}\left(M_{a}\right)=\mathbb{Z}\left[u_{1}, \ldots, u_{4}, x\right] /\left\langle u_{2} u_{3}, u_{1}\left(x-u_{2}\right) u_{4}, u_{1} u_{3} u_{4}\right\rangle, \\
& H_{T^{d} \times S^{1}}^{*}\left(M_{b}\right)=\mathbb{Z}\left[u_{1}, \ldots, u_{4}, x\right] /\left\langle\left(x-u_{2}\right) u_{3}, u_{1} u_{2} u_{4}, u_{1} u_{3} u_{4}\right\rangle, \\
& H_{T^{d} \times S^{1}}^{*}\left(M_{c}\right)=\mathbb{Z}\left[u_{1}, \ldots, u_{4}, x\right] /\left\langle u_{2} u_{3},\left(x-u_{1}\right) u_{2}\left(x-u_{4}\right), u_{1} u_{3} u_{4}\right\rangle .
\end{aligned}
$$

As we have already observed, $H_{T^{d} \times S^{1}}^{*}\left(M_{a}\right)$ and $H_{T^{d} \times S^{1}}^{*}\left(M_{b}\right)$ are isomorphic by interchanging $u_{2}$ with $x-u_{2}$. One can check that the annihilator of $u_{2}$ in $H_{T^{d} \times S^{1}}^{*}\left(M_{a}\right)$ is the principal ideal generated by $u_{3}$, while the ring $H_{T^{d} \times S^{1}}^{*}\left(M_{c}\right)$ has no degree 2 element whose annihilator is generated by a single element of degree 2 . Hence $H_{T^{d} \times S^{1}}^{*}\left(M_{c}\right)$ is not isomorphic to the other two rings.

The ring $H_{S^{1}}^{*}(M)$, on the other hand, is sensitive to coorientations as well as the affine structure of $\mathcal{H}$.

Example 4.11. We now compute the ring $H_{S^{1}}^{*}(M)$ for $M_{a}, M_{b}$, and $M_{c}$ of Figure 2. Theorem 4.5 tells us that we need only to quotient the ring $H_{T^{d} \times S^{1}}^{*}(M)$ by $\operatorname{ker}\left(\iota^{*}\right)$. For $M_{a}$, the kernel of $\iota_{a}^{*}$ is generated by $u_{1}+u_{2}-u_{3}$ and $u_{1}-u_{4}$, hence we have

$$
\begin{aligned}
H_{S^{1}}^{*}\left(M_{a}\right) & =\mathbb{Z}\left[u_{2}, u_{3}, x\right] /\left\langle u_{2} u_{3},\left(u_{3}-u_{2}\right)^{2}\left(x-u_{2}\right),\left(u_{3}-u_{2}\right)^{2} u_{3}\right\rangle \\
& \cong \mathbb{Z}\left[u_{2}, u_{3}, x\right] /\left\langle u_{2} u_{3},\left(u_{3}-u_{2}\right)^{2}\left(x-u_{2}\right), u_{3}^{3}\right\rangle .
\end{aligned}
$$

Since the hyperplanes of 2(c) have the same coorientations as those of 2(a), we have $\operatorname{ker} \iota_{b}^{*}=\operatorname{ker} \iota_{a}^{*}$, hence

$$
\begin{aligned}
H_{S^{1}}^{*}\left(M_{c}\right) & =\mathbb{Z}\left[u_{2}, u_{3}, x\right] /\left\langle u_{2} u_{3},\left(x-u_{3}+u_{2}\right)^{2} u_{2},\left(u_{3}-u_{2}\right)^{2} u_{3}\right\rangle \\
& \cong \mathbb{Z}\left[u_{2}, u_{3}, x\right] /\left\langle u_{2} u_{3},\left(x-u_{3}+u_{2}\right)^{2} u_{2}, u_{3}^{3}\right\rangle .
\end{aligned}
$$

Finally, since Figure 2(b) is obtained from 2(a) by flipping the coorientation of $H_{2}$, we find that $\operatorname{ker}\left(\iota_{b}^{*}\right)$ is generated by $u_{1}-u_{2}-u_{3}$ and $u_{1}-u_{4}$, therefore

$$
H_{S^{1}}^{*}\left(M_{b}\right)=\mathbb{Z}\left[u_{2}, u_{3}, x\right] /\left\langle\left(x-u_{2}\right) u_{3},\left(u_{2}+u_{3}\right)^{2} u_{2},\left(u_{2}+u_{3}\right)^{2} u_{3}\right\rangle .
$$

As in Example 4.10, $H_{S^{1}}^{*}\left(M_{a}\right)$ and $H_{S^{1}}^{*}\left(M_{c}\right)$ can be distinguished by the fact that the annihilator of $u_{2} \in H_{S^{1}}^{*}\left(M_{a}\right)$ is generated by a single element of degree 2, and no element of $H_{S^{1}}^{*}\left(M_{c}\right)$ has this property. On the other hand, $H_{S^{1}}^{*}\left(M_{b}\right)$ is distinguished from $H_{S^{1}}^{*}\left(M_{a}\right)$ and $H_{S^{1}}^{*}\left(M_{c}\right)$ by the fact that neither $x-u_{2}$ nor $u_{3}$ cubes to zero. 
Remark 4.12. Theorems 4.4 and 4.5 can be interpreted in light of the recent work of Hausel and Sturmfels [HS] on Lawrence toric varieties. The Lawrence toric variety $N$ associated to the arrangement $\mathcal{H}$ is the Kähler reduction $T^{*} \mathbb{C}^{n} / / T^{k}$, so that $M$ sits inside of $N$ as the complete intersection cut out by the equation $\overline{\mu_{\mathbb{C}}}(z, w)=0$. The residual torus acting on $N$ has dimension $d+n$, and includes the $(d+1)$-dimensional torus $T^{d} \times S^{1}$ acting on $M$, and the inclusion of $M$ into $N$ induces an isomorphism on $T^{d} \times S^{1}$-equivariant cohomology. One can use geometric arguments similar to those that were applied to prove Theorem 4.4, or the purely combinatorial approach of $[\mathbf{H S}]$, to show that

$$
H_{T^{d+n}}^{*}(N)=\mathbb{Q}\left[u_{1}, \ldots, u_{n}, v_{1}, \ldots, v_{n}\right] /\left\langle\prod_{i \in S_{1}} u_{i} \times \prod_{j \in S_{2}} v_{j} \mid \bigcap_{i \in S} H_{i}=\emptyset\right\rangle .
$$

From here we can recover $H_{T^{d} \times S^{1}}^{*}(M)=H_{T^{d} \times S^{1}}^{*}(N)$ by setting $u_{i}+v_{i}=$ $u_{j}+v_{j}$ for all $i, j \leq n$. Note that Hausel and Sturmfels' work applies to the general orbifold case.

\section{A deformation of the Orlik-Solomon algebra of $\mathcal{H}$.}

Let $M_{\mathbb{R}} \subseteq M$ be the real locus $\{[z, w] \in M \mid z, w$ real $\}$ of $M$ with respect to the complex structure $J_{1}$. The full group $T^{d} \times S^{1}$ does not act on $M_{\mathbb{R}}$, but the subgroup $T_{\mathbb{R}}^{d} \times \mathbb{Z}_{2}$ does act, where $T_{\mathbb{R}}^{d}:=\mathbb{Z}_{2}^{d} \subseteq T^{d}$ is the fixed point set of the involution of $T^{d}$ given by complex conjugation. ${ }^{3}$ In this section we will study the geometry of the real locus, focusing in particular on the properties of the residual $\mathbb{Z}_{2}$ action.

A proof of a more general statement of the following theorem is forthcoming in $[\mathbf{H H}]$ :

Theorem 5.1. Let $G=T^{d} \times S^{1}$ or $T^{d}$, and $G_{\mathbb{R}}=T_{\mathbb{R}}^{d} \times \mathbb{Z}_{2}$ or $T_{\mathbb{R}}^{d}$. Then we have $H_{G}^{2 *}\left(M ; \mathbb{Z}_{2}\right) \cong H_{G_{\mathbb{R}}}^{*}\left(M_{\mathbb{R}} ; \mathbb{Z}_{2}\right)$, i.e., the rings are isomorphic by an isomorphism that halves the grading. Furthermore, this isomorphism identifies the class $u_{i} \in H_{G}^{*}\left(M ; \mathbb{Z}_{2}\right)$, represented by the divisor $Z_{i}$, with the class in $H_{G_{\mathbb{R}}}^{*}\left(M_{\mathbb{R}} ; \mathbb{Z}_{2}\right)$ represented by the divisor $Z_{i} \cap M_{\mathbb{R}}$, and likewise takes $x-u_{i}$ (if $\left.G=T^{d} \times S^{1}\right)$ or $-u_{i}\left(\right.$ if $\left.G=T^{d}\right)$ to the class represented by $W_{i} \cap M_{\mathbb{R}}$.

Sketch of proof. Consider the injection $H_{G}^{*}\left(M ; \mathbb{Z}_{2}\right) \hookrightarrow H_{G}^{*}\left(M^{G} ; \mathbb{Z}_{2}\right)$ given by the inclusion of the fixed point set into $M$. The essential idea is to show that a class in $H_{G}^{*}\left(M^{G} ; \mathbb{Z}_{2}\right)$ extends over $M$ if and only if it extends to the set of points on which $G$ acts with a stabilizer of codimension at most 1, and

\footnotetext{
${ }^{3}$ It is interesting to note that the real locus with respect to the complex structure $J_{1}$ is in fact a complex submanifold with respect to the one of the other complex structures on $M$. The action of $T_{\mathbb{R}}^{d}$ is holomorphic because $T_{\mathbb{R}}^{d}$ is a subgroup of $T^{d}$, which preserves all of the complex structures on $M$. The action of $\mathbb{Z}_{2}$, on the other hand, is anti-holomorphic, i.e., it can be thought of as complex conjugation.
} 
then to show that a similar statement in $G_{\mathbb{R}^{-} \text {-equivariant cohomology also }}$ holds for the real locus $M_{\mathbb{R}}$ with its $G_{\mathbb{R}}$ action. One then uses a canonical isomorphism $H_{G}^{2}\left(p t, \mathbb{Z}_{2}\right) \cong H_{G_{\mathbb{R}}}^{1}\left(p t, \mathbb{Z}_{2}\right)$ to give the result.

The key to the proof is a noncompact $G_{\mathbb{R}}$ version of the proposition in [TW] stating that the $G_{\mathbb{R}}$-equivariant Euler class of the negative normal bundle of a critical point $p$ is not a zero divisor, which can be shown explicitly using a local normal form for the actions of $G$ and $G_{\mathbb{R}}$. The proposition then follows from standard $G_{\mathbb{R}}$ versions of the Thom isomorphism theorem with coefficients in $\mathbb{Z}_{2}$. Since a component of the moment map is proper, bounded below, and has finitely many fixed points, one can then check that the inductive argument, given in Section 3 of $[\mathbf{T W}]$ to complete the proof of [ $\mathbf{T W}$, Thm 1] also holds in this case.

Let us consider the restriction of the hyperkähler moment map $\mu_{\mathrm{HK}}=$ $\mu_{\mathbb{R}} \oplus \mu_{\mathbb{C}}$ to $M_{\mathbb{R}}$. Since $z$ and $w$ are real for every $[z, w] \in M_{\mathbb{R}}$, the map $\mu_{\mathbb{C}}$ takes values in $\mathfrak{t}_{\mathbb{R}}^{d} \subseteq \mathfrak{t}_{\mathbb{C}}^{d}$, which we will identify with $i \mathbb{R}^{n}$, so that $f=$ $\left.\left.\mu_{\mathbb{R}}\right|_{M_{\mathbb{R}}} \oplus \mu_{\mathbb{C}}\right|_{M_{\mathbb{R}}}$ takes values in $\mathbb{R}^{n} \oplus i \mathbb{R}^{n} \cong \mathbb{C}^{n}$. Note that $f$ is $\mathbb{Z}_{2}$-equivariant, with $\mathbb{Z}_{2}$ acting on $\mathbb{C}^{n}$ by complex conjugation.

Lemma 5.2. The map $f: M_{\mathbb{R}} \rightarrow \mathbb{C}^{n}$ is surjective, and the fibers are the orbits of $T_{\mathbb{R}}^{d}$. The stabilizer of a point $x \in M_{\mathbb{R}}$ has order $2^{r}$, where $r$ is the number of hyperplanes in the complexified arrangement $\mathcal{H}_{\mathbb{C}}$ containing the point $f(x)$.

Proof. For any point $p=a+b i \in \mathbb{C}^{n}$, choose a point $[z, w] \in M$ such that $\mu_{\mathbb{R}}[z, w]=a$ and $\mu_{\mathbb{C}}[z, w]=b$. We can hit $[z, w]$ with an element of $T^{d}=T^{n} / T^{k}$ to make $z$ real, and the fact that $\mu_{\mathbb{C}}[z, w] \in \mathbb{R}^{n}$ forces $w$ to be real as well, hence we may assume that $[z, w] \in M_{\mathbb{R}}$. Then $f^{-1}(p)=$ $\mu_{\mathrm{HK}}^{-1}(a, b) \cap M_{\mathbb{R}}=T^{d}[z, w] \cap M_{\mathbb{R}}=T_{\mathbb{R}}^{d}[z, w]$. The second statement follows easily from $[\mathbf{B D}, 3.1]$.

Let $Y \subseteq M_{\mathbb{R}}$ be the locus of points on which $T_{\mathbb{R}}^{d}$ acts freely, i.e., the preimage under $f$ of the space $\mathcal{M}(\mathcal{H}):=\mathbb{C}^{n} \backslash \cup_{i=1}^{n} H_{i}^{\mathbb{C}}$. The inclusion map $Y \hookrightarrow M_{\mathbb{R}}$ induces maps backward on cohomology, which we will denote

$$
\phi: H_{T_{\mathbb{R}}^{d}}^{*}\left(M_{\mathbb{R}} ; \mathbb{Z}_{2}\right) \rightarrow H_{T_{\mathbb{R}}^{d}}^{*}\left(Y ; \mathbb{Z}_{2}\right) \cong H^{*}\left(\mathcal{M}(\mathcal{H}) ; \mathbb{Z}_{2}\right)
$$

and

$$
\phi_{2}: H_{T_{\mathbb{R}}^{d} \times \mathbb{Z}_{2}}^{*}\left(M_{\mathbb{R}} ; \mathbb{Z}_{2}\right) \rightarrow H_{T_{\mathbb{R}}^{d} \times \mathbb{Z}_{2}}^{*}\left(Y ; \mathbb{Z}_{2}\right) \cong H_{\mathbb{Z}_{2}}^{*}\left(\mathcal{M}(\mathcal{H}) ; \mathbb{Z}_{2}\right) .
$$

The ring $H^{*}(\mathcal{M}(\mathcal{H}) ; \mathbb{Z})$ has been studied extensively, and is called the OrlikSolomon algebra $[\mathbf{O T}]$, which we will denote by $\mathcal{O S}$. A remarkable fact about the Orlik-Solomon algebra is that it depends only on the combinatorial structure of $\mathcal{H}$; the following is a presentation in terms of anticommuting generators $e_{1}, \ldots, e_{n}[\mathbf{O S}]$ :

$$
\mathcal{O S} \cong H^{*}(\mathcal{M}(\mathcal{H}) ; \mathbb{Z}) \cong \mathbb{Z}\left[e_{1}, \ldots, e_{n}\right] /\left\langle\Pi_{i \in S} e_{i} \mid \cap_{i \in S} H_{i}=\emptyset\right\rangle
$$


Rather than working with anticommuting generators, we can work over the ground field $\mathbb{Z}_{2}$, in which case commutativity and anticommutativity are the same. Because $\mathcal{O S}$ is torsion-free [OT, 3.74], we have

$\mathcal{O S} \otimes \mathbb{Z}_{2} \cong H^{*}\left(\mathcal{M}(\mathcal{H}) ; \mathbb{Z}_{2}\right)$

$$
\cong \mathbb{Z}_{2}\left[e_{1}, \ldots, e_{n}\right] /\left\langle\Pi_{i \in S} e_{i} \mid \cap_{i \in S} H_{i}=\emptyset\right\rangle+\left\langle e_{i}^{2} \mid i \leq n\right\rangle,
$$

where $\operatorname{deg}\left(e_{i}\right)=1$.

Claim 5.3. The map $\phi: H_{T_{\mathbb{R}}^{d}}^{*}\left(M_{\mathbb{R}} ; \mathbb{Z}_{2}\right) \rightarrow \mathcal{O S} \otimes \mathbb{Z}_{2}$ takes $u_{i}$ to $e_{i}$, hence ker $\phi$ is generated by the set $\left\{u_{i}^{2} \mid i \leq n\right\}$.

Proof. Recall from Section 2 that the hyperplane $H_{i} \subseteq\left(\mathfrak{t}^{d}\right)^{*}$ is defined by the equation $\left\langle x, a_{i}\right\rangle=\left\langle-\widetilde{\alpha}, \varepsilon_{i}\right\rangle$. Let $\eta_{i}: \mathbb{C}^{n} \rightarrow \mathbb{C}$ be the affine map taking $x$ to $\left\langle x, a_{i}\right\rangle+\left\langle\widetilde{\alpha}, \varepsilon_{i}\right\rangle$, so that $H_{i}^{\mathbb{C}}$ is cut out of $\mathbb{C}^{n}$ by $\eta_{i}$. Then $\eta_{i}$ restricts to a map $\mathcal{M}(\mathcal{H}) \rightarrow \mathbb{C}^{*}$, and Orlik and Terao identify $e_{i}$ with the cohomology class represented by the pull-back of the submanifold $\mathbb{R}_{-}$(the negative reals) along $\eta_{i}$ [OT, 5.90]. Theorem 5.1 tells us that the cohomology class $u_{i}$ is represented by the divisor $Z_{i} \cap M_{\mathbb{R}}$. By Lemma 3.1, $f\left(Z_{i} \cap M_{\mathbb{R}}\right)=G_{i} \cap \mathbb{R}^{n}$, hence $\phi\left(u_{i}\right)$ is the class represented by the submanifold $G_{i} \cap \mathbb{R}^{n} \cap \mathcal{M}(\mathcal{H})=$ $\eta_{i}^{-1}\left(\mathbb{R}_{-}\right)$.

Remark 5.4. The fact that the classes $u_{i}^{2}$ lie in the kernel of $\phi$ can be seen by noting that $u_{i}$ is represented both by the divisor

$$
Z_{i}=\left\{[z, w] \in M_{\mathbb{R}} \mid z_{i}=0\right\},
$$

and, since $u_{i}=-u_{i}$ over $\mathbb{Z}_{2}$, also by the divisor

$$
W_{i}=\left\{[z, w] \in M_{\mathbb{R}} \mid w_{i}=0\right\} .
$$

The condition $x \in Z_{i} \cap W_{i}$ says exactly that $\mu_{\mathbb{R}}(x) \in H_{i}$, therefore the intersection $Z_{i} \cap W_{i} \cap Y$ is empty.

In some sense we have cheated here; we have concluded that we can recover a presentation of $\mathcal{O S} \otimes \mathbb{Z}_{2}$ from a presentation of $H_{T^{d}}^{*}(M)$, but we used the fact that we already have a presentation of $\mathcal{O S} \otimes \mathbb{Z}_{2}$. In the $\mathbb{Z}_{2^{-}}$ equivariant picture, however, our trivial observation turns magically into new information, giving us a presentation of the equivariant cohomology $\operatorname{ring} H_{\mathbb{Z}_{2}}^{*}\left(\mathcal{M}(\mathcal{H}) ; \mathbb{Z}_{2}\right)$.

Theorem 5.5. The map $\phi_{2}$ is surjective, with kernel generated by $\left\{u_{i}(x-\right.$ $\left.\left.u_{i}\right) \mid i \leq n\right\}$. Hence

$$
\begin{aligned}
& H_{\mathbb{Z}_{2}}^{*}\left(\mathcal{M}(\mathcal{H}) ; \mathbb{Z}_{2}\right) \cong H_{T_{\mathbb{R}}^{d} \times \mathbb{Z}_{2}}^{*}\left(M_{\mathbb{R}} ; \mathbb{Z}_{2}\right) / \operatorname{ker} \phi_{2} \\
& \quad \cong \frac{\mathbb{Z}_{2}\left[u_{1}, \ldots, u_{n}, x\right]}{\left\langle\Pi_{i \in S_{1}} u_{i} \times \Pi_{j \in S_{2}}\left(x-u_{j}\right) \mid \cap_{i \in S} H_{i}=\emptyset\right\rangle+\left\langle u_{i}\left(x-u_{i}\right) \mid i \leq n\right\rangle} .
\end{aligned}
$$


Proof. The fact that $\mathcal{H}_{\mathbb{C}}$ is the complexification of a real arrangement tells us that the linear forms $\eta_{i}$ have real coefficients, therefore the generators $e_{i}$ of $H^{*}\left(\mathcal{M}(\mathcal{H}) ; \mathbb{Z}_{2}\right)$ are $\mathbb{Z}_{2}$-invariant. This implies, by [Bo, 3.5], that $H_{\mathbb{Z}_{2}}^{*}\left(\mathcal{M}(\mathcal{H}) ; \mathbb{Z}_{2}\right)$ is a free module over $H_{\mathbb{Z}_{2}}^{*}(p t)$. Then surjectivity of $\phi_{2}$ follows from surjectivity of $\phi$ using a formal argument identical to that of Corollary 4.9. By Theorem 5.1 and Proposition $4.7, H_{T_{\mathbb{R}}^{d} \times \mathbb{Z}_{2}}^{*}\left(M_{\mathbb{R}} ; \mathbb{Z}_{2}\right)$ is a free module over $H_{\mathbb{Z}_{2}}^{*}(p t)$, therefore $\operatorname{ker} \phi_{2}$ is a free $H_{\mathbb{Z}_{2}}^{*}(p t)$-module of rank $n$. The fact that $u_{i}\left(x-u_{i}\right) \in \operatorname{ker} \phi_{2}$ follows from the argument of Remark 5.4, hence we are done.

The ring $H_{\mathbb{Z}_{2}}^{*}\left(\mathcal{M}(\mathcal{H}) ; \mathbb{Z}_{2}\right)$ is therefore a deformation of $\mathcal{O S} \otimes \mathbb{Z}_{2}$ (over the base Spec $\left.\mathbb{Z}_{2}[x]\right)$ that depends nontrivially on the affine structure of $\mathcal{H}$, rather than simply on the underlying matroid.

Example 5.6. Consider the arrangements $\mathcal{H}_{a}$ and $\mathcal{H}_{c}$ in Figure 2(a) and 2(c). By Theorem 5.5 and Example 4.10 we have

$H_{\mathbb{Z}_{2}}^{*}\left(\mathcal{M}\left(\mathcal{H}_{a}\right) ; \mathbb{Z}_{2}\right) \cong \mathbb{Z}_{2}\left[u_{1}, \ldots, u_{4}, x\right] /\left\langle\begin{array}{c}u_{1}\left(x-u_{1}\right), u_{2}\left(x-u_{2}\right), u_{3}\left(x-u_{3}\right), u_{4}\left(x-u_{4}\right), \\ u_{2} u_{3}, u_{1}\left(x-u_{2}\right) u_{4}, u_{1} u_{3} u_{4}\end{array}\right\rangle$ and

$H_{\mathbb{Z}_{2}}^{*}\left(\mathcal{M}\left(\mathcal{H}_{c}\right) ; \mathbb{Z}_{2}\right) \cong \mathbb{Z}_{2}\left[u_{1}, \ldots, u_{4}, x\right] /\left\langle\begin{array}{c}u_{1}\left(x-u_{1}\right), u_{2}\left(x-u_{2}\right), u_{3}\left(x-u_{3}\right), u_{4}\left(x-u_{4}\right), \\ u_{2} u_{3},\left(x-u_{1}\right) u_{2}\left(x-u_{4}\right), u_{1} u_{3} u_{4}\end{array}\right\rangle$.

The map $f: H_{\mathbb{Z}_{2}}^{*}\left(\mathcal{M}\left(\mathcal{H}_{a}\right) ; \mathbb{Z}_{2}\right) \rightarrow H_{\mathbb{Z}_{2}}^{*}\left(\mathcal{M}\left(\mathcal{H}_{b}\right) ; \mathbb{Z}_{2}\right)$ given by

$$
\begin{gathered}
f\left(u_{1}\right)=u_{1}+u_{2}, \quad f\left(u_{2}\right)=u_{2}+u_{3}+x, \\
f\left(u_{3}\right)=u_{3}, \quad f\left(u_{4}\right)=u_{2}+u_{4}, \text { and } f(x)=x
\end{gathered}
$$

is an isomorphism of graded $\mathbb{Z}_{2}[x]$-algebras, hence the ring $H_{\mathbb{Z}_{2}}^{*}\left(\mathcal{M}(\mathcal{H}) ; \mathbb{Z}_{2}\right)$ is not a complete invariant of smooth, rational, affine arrangements up to combinatorial equivalence. ${ }^{4}$

Example 5.7. Now consider the arrangements $\mathcal{H}_{a}^{\prime}$ and $\mathcal{H}_{c}^{\prime}$ obtained from $\mathcal{H}_{a}$ and $\mathcal{H}_{c}$ by adding a vertical line on the far left, as shown below.

Again by Theorem 5.5, we have

$$
H_{\mathbb{Z}_{2}}^{*}\left(\mathcal{M}\left(\mathcal{H}_{a}^{\prime}\right) ; \mathbb{Z}_{2}\right) \cong \mathbb{Z}_{2}[\vec{u}, x] /\left\langle\begin{array}{c}
u_{1}\left(x-u_{1}\right), u_{2}\left(x-u_{2}\right), u_{3}\left(x-u_{3}\right), u_{4}\left(x-u_{4}\right), \\
u_{5}\left(x-u_{5}\right), u_{2} u_{3},\left(x-u_{1}\right) u_{5}, u_{1}\left(x-u_{2}\right) u_{4}, \\
u_{1} u_{3} u_{4},\left(x-u_{2}\right) u_{4} u_{5}, u_{3} u_{4} u_{5}
\end{array}\right\rangle
$$

and

$$
H_{\mathbb{Z}_{2}}^{*}\left(\mathcal{M}\left(\mathcal{H}_{c}^{\prime}\right) ; \mathbb{Z}_{2}\right) \cong \mathbb{Z}_{2}[\vec{u}, x] /\left\langle\begin{array}{c}
u_{1}\left(x-u_{1}\right), u_{2}\left(x-u_{2}\right), u_{3}\left(x-u_{3}\right), u_{4}\left(x-u_{4}\right), \\
u_{5}\left(x-u_{5}\right), u_{2} u_{3},\left(x-u_{1}\right) u_{5},\left(x-u_{1}\right) u_{2}\left(x-u_{4}\right), \\
u_{1} u_{3} u_{4},\left(x-u_{2}\right) u_{4} u_{5}, u_{3} u_{4} u_{5}
\end{array}\right\rangle .
$$

We have used Macaulay 2 to check that the annihilator of the element $u_{2} \in H_{\mathbb{Z}_{2}}^{*}\left(\mathcal{M}\left(\mathcal{H}_{a}^{\prime}\right) ; \mathbb{Z}_{2}\right)$ is generated by two linear elements (namely $u_{3}$ and $\left.x-u_{2}\right)$ and nothing else, while there is no element of $H_{\mathbb{Z}_{2}}^{*}\left(\mathcal{M}\left(\mathcal{H}_{c}^{\prime}\right) ; \mathbb{Z}_{2}\right)$ with this property. Hence the two rings are not isomorphic.

\footnotetext{
${ }^{4}$ We thank Graham Denham for finding this isomorphism.
} 
$\mathcal{H}_{a}^{\prime}$

$\mathcal{H}_{c}^{\prime}$

Acknowledgments. We are very grateful to Tamás Hausel for introducing us to this problem and answering many questions. We would also like to thank Allen Knutson for guiding our research.

\section{References}

[BD] R. Bielawski and A. Dancer, The geometry and topology of toric hyperkähler manifolds, Comm. Anal. Geom., 8 (2000), 727-760, MR 1792372, Zbl 0992.53034.

[BGH] D. Biss, T. Holm and V. Guillemin, The mod 2 cohomology of fixed point sets of anti-symplectic involutions, math.SG/0107151.

[Bo] A. Borel, Seminar on Transformation Groups, Chapter XII. Annals of Mathematics Studies, 46, Princeton, 1960, MR 0116341, Zbl 0091.37202.

[BJ] T. Brocker and K. Janich, Introduction to Differential Topology, Cambridge University Press, 1982, MR 0674117.

[HH] M. Harada and T. Holm, The equivariant cohomology of hypertoric varieties and their real loci, in preparation.

[HS] T. Hausel and B. Sturmfels, Toric hyperkähler varieties, math.AG/0203096.

[HL] P. Heinzner and F. Loose, Reduction of complex Hamiltonian G-spaces, Geom. Funct. Anal., 4(3) (1994), 288-297, MR 1274117, Zbl 0816.53018.

[HKLR] N. Hitchin, A. Karlhede, U. Lindström and M. Roček, Hyper-Kähler metrics and supersymmetry, Comm. Math. Phys., 108(4) (1987), 535-589, MR 0877637, Zbl 0612.53043.

[Ki] F. Kirwan, Cohomology of Quotients in Symplectic and Algebraic Geometry, Mathematical Notes, 31, Princeton University Press, 1984, MR 0766741, Zbl 0553.14020.

[K1] H. Konno, Cohomology rings of toric hyperkähler manifolds, Internat. J. of Math., 11(8) (2000), 1001-1026, MR 1797675, Zbl 0991.53027.

[K2] Equivariant cohomology rings of toric hyperkähler manifolds, in 'Quaternionic structures in mathematics and physics (Rome, 1999)', 231-240 (electronic), MR 1848663, Zbl 0991.53028.

[Na] H. Nakajima, Instantons on ALE spaces, quiver varieties, and Kac-Moody algebras, Duke Math. J., 76(2) (1994), 365-416, MR 1302318, Zbl 0826.17026. 
[OS] P. Orlik and L. Solomon, Combinatorics and topology of complements of hyperplanes, Invent. Math., 56 (1980), 167-189, MR 0558866, Zbl 0432.14016.

[OT] P. Orlik and H. Terao, Arrangements of Hyperplanes, Grundlehren der mathematischen Wissenschaften, 300, Springer-Verlag, 1992, MR 1217488, Zbl 0757.55001.

[Pr] N. Proudfoot, The equivariant Orlik-Solomon algebra, http://math.berkeley.edu/ ${ }^{\sim}$ proudf/os.ps.

[Sc] C. Schmid, Cohomologie Équivariante des Certaines Variétés Hamiltoniennes et de leur Partie Réelle, Thèse à Université de Genève, http://www.unige.ch/math/biblio/these/theses.html.

[Sj] R. Sjamaar, Holomorphic slices, symplectic reduction, and multiplicities of representations, Ann. of Math., 141 (1995), 87-129, MR 1314032, Zbl 0827.32030.

[TW] S. Tolman and J. Weitsman, On the cohomology rings of Hamiltonian T-spaces, in 'Proc. of the Northern California Symplectic Geometry Seminar', Y. Eliashberg et al. eds., Amer. Math. Soc. Transl. Ser. 2, 196, Advances in Mathematical Sciences, 45 (1999), 251-258, MR 1736221, Zbl 0955.57023.

Department of Mathematics

UNIVERSITY OF CALIFORNIA

BERKELEY, CA 94720

E-mail address: megumi@math.berkeley.edu

Department of Mathematics

University of CALIFORNIA

Berkeley, CA 94720

E-mail address: proudf@math.berkeley.edu 